\title{
MODELOS MENTALES Y APRENDIZAJE DE FÍSICA EN ELECTRICIDAD Y MAGNETISMO
}

GRECA, I.M. y MOREIRA, M.A. Instituto de Física, UFRGS. Caixa Postal, 15051. Campus do Vale. Porto Alegre, RS. Brasil.

\begin{abstract}
SUMMARY
A study conducted under the framework of Johnson-Laird's mental model theory is described. The objective of such a study was an attempt to categorize introductory college physics students according to the type of mental representations - propositions, images, and models- they used when solving problems and conceptual questions in electricity and magnetism. The basic features of each category are presented and several examples are given. At the end implications for research and instruction are discussed.
\end{abstract}

\section{INTRODUCCIÓN}

Hoy es un consenso que la mente de las personas no es una hoja en blanco en donde pueden ser colocadas informaciones arbitrariamente. Por algún tipo de mecanismo, cualquier información nueva que una persona recibe interactúa con aquello que ya sabe, y el producto de esa interacción, resultante en nuevos significados, podría ser definido como aprendizaje. Es por esta razón que conocer cómo las personas representan internamente el mundo en que viven resulta esencial tanto para saber lo que es la cognición como para la elaboración de estrategias instruccionales que faciliten el aprendizaje.

Cómo representamos exactamente el mundo en nuestras cabezas ha sido la gran pregunta de la filosofía, la psicología y la lingüística durante siglos. Una representación puede ser definida como cualquier notación, signo o conjunto de símbolos que «representan» algo para nosotros (Eisenck y Keane, 1991). Está en lugar de alguna cosa, en su ausencia; habitualmente es un aspecto del mundo externo o de nuestra imaginación. Una teoría científica es, en estos términos, un sistema «representa- cional», externamente representado por su formulación matemática e internamente, de alguna a manera en la cabeza de quien la comprende. Para la enseñanza de la física resulta interesante preguntarse cuáles son las representaciones internas que los alumnos tienen -tanto las que corresponden a los conceptos «intuitivos» como las que construyen a partir de los conceptos enseñados en el aula- para poder entender cuál es el proceso de construcción y el cambio de esas representaciones. El trabajo que presentamos aquí tuvo por objetivo justamente determinar a qué nivel de representación mental operan los estudiantes de física general de nivel universitario cuando resuelven problemas y cuestiones teóricas referidas al concepto de campo electromagnético.

Varias formas de representación han sido propuestas por los psicólogos cognitivos. Aunque sea punto de controversia si existen otras formas representacionales que no sean cadenas de símbolos, la base teórica de este trabajo se encuadra dentro de la teoría de los modelos mentales de Johnson-Laird (1983), para quien existen por lo 
menos tres: $a$ ) las representaciones proposicionales (definidas como representaciones mentales que pueden ser expresadas verbalmente); b) los modelos mentales (análogos estructurales de una situación del mundo real o imaginario); c) las imágenes mentales («perspectivas» particulares de un modelo mental).

Aunque el nivel de codificación interna de todas las representaciones sea proposicional ${ }^{1}$, para Johnson-Laird, el punto central del razonamiento y de la comprensión de cualquier fenómeno, evento, situación o proceso del mundo real o imaginario está en la existencia de un modelo de trabajo en la mente de quien razona y comprende. Pensar, entonces, en términos de una teoría científica implica ser la construcción de un modelo mental. En cuanto a los conceptos científicos, pueden ser, y de hecho la mayoría de las veces son, codificados proposicionalmente, y comprenderlos implica construir modelos mentales de los procesos que representan.

Los modelos mentales que pueden ser construidos como resultado de la percepción, interacción social o experiencia interna permiten a los individuos hacer inferencias y predicciones, entender los fenómenos, decidir las acciones a tomar y controlar su ejecución. Éste es el sentido del working model.

Según la teoría de los modelos mentales, no es preciso postular que las personas tenemos internalizadas una lógica para poder realizar inferencias, sino que para ello es necesario representar internamente situaciones concretas -de mundos reales o imaginarios- y evaluar, en función de ellos, la validez del nuestro razonamiento. Esta postura frente al mecanismo del razonamiento intenta dar cuenta tanto de las serias dificultades que enfrentan las teorías que postulan el razonamiento basado en la lógica formal (por ejemplo, la escuela de Ginebra) como de la fuerte influencia que tiene el contenido de las premisas en nuestras inferencias. Así, es posible pensar que, cuando cometemos errores en nuestras inferencias -lo que ocurre frecuentemente-, es debido a no haber puesto a prueba los modelos que creamos. La hipótesis de que realizamos inferencias vía modelos mentales gana plausibilidad cuando consideramos que, como seres vivos, debemos adaptarnos a un medio en continuo cambio (Nersessian, 1992). Los investigadores en inteligencia artificial se enfrentan a grandes dificultades cuando los sistemas de representación de conocimiento, proposicionalmente representados, sufren pequeños cambios.

Las tres formas de representaciones -representaciones proposicionales, modelos mentales e imágenes- se encuentran relacionadas. Las representaciones proposicionales son interpretadas a la luz de los modelos mentales que se poseen. Serán evaluadas como verdaderas en la medida que puedan ser inferidas de los modelos disponibles. Las imágenes son «señales» del modelo. Así cuando imaginamos un triángulo, lo hacemos de uno específico equilátero, rectángulo, etc. Sin embargo, el modelo subyacente debe contener las relaciones necesarias para definir un triángulo en general, de manera tal que, si nos fuera pedido que imaginásemos un triángulo escaleno, podríamos hacerlo. Por otra parte, si se afirmase que la «suma de los ángulos interiores de un triángulo es igual a $270^{\circ} \gg$, deberíamos ser capaces de evaluarla con respecto al modelo, pudiendo entonces decir si es falsa o verdadera (Greca y Moreira, 1996).

\section{MODELOS MENTALES Y APRENDIZAJES EN FISICA}

Cuando entendemos un fenómeno físico, sabemos cuál es su causa y resultado, sabemos como iniciarlo, influenciarlo o evitarlo; en el lenguaje de Johnson-Laird, es tener un modelo de trabajo de ese fenómeno (Greca, 1995).

En los alumnos, la comprensión de los conceptos, proposiciones (formulaciones matemáticas, definiciones) analogías y procedimientos experimentales dependerá de la formación de modelos mentales. Los alumnos traen al aula los modelos mentales con los cuales ya entendían, imaginaban, explicaban el mundo antes de ir a la escuela. En situaciones donde no existe un profesor, las personas construyen modelos de los fenómenos físicos por sí mismas, para comprender ese mundo. Juntamente con la percepción, que es la fuente principal de construcción de modelos, las personas utilizan en general tres principios (Johnson-Laird, 1990):

1) en el dominio determinista, todos los eventos tienen causa;

2) las causas preceden a los eventos;

3) la acción sobre un objeto es la principal causa para cualquier cambio que ocurra en él.

Con estos elementos es posible construir modelos causales simples del sistema físico. Los modelos llevan implícitos mecanismos de construcción, de realización de inferencias que, básicamente, consistirían en la visualización de situaciones, en el «rodaje» del modelo y en la lectura directa de los resultados, sin la mediación de mecanismos lógicos. Estos mecanismos serían independientes del modelo usado. Por tanto, más que concepciones alternativas aisladas, son formas de entender el mundo.

La clasificación de Pozo y otros (1994) para el origen de las concepciones alternativas puede servir también para clasificar los modelos mentales formados por los alumnos. Los de origen sensorial corresponderían a lo que consideramos la fuente principal de modelos mentales, la percepción; los de origen cultural serían los modelos mentales inducidos por el contexto social de los alumnos (por ejemplo, los grupos culturales regidos por religiones que dan explicaciones a los fenómenos derivadas de las intenciones o deseos de seres sobrenaturales construirán modelos mentales distintos que los de los grupos de sociedades tecnologizadas); y los de origen escolar, 
aquéllos generados dentro del contexto escolar en temas sobre los cuales el alumno carece de conocimientos específicos (como la mecánica cuántica), por analogías propias o inducidas por la enseñanza.

En resumen, la capacidad de entender una teoría científica estará determinada por la capacidad del alumno para formar modelos que incluyan las relaciones fundamentales de la teoría y de los cuales sea posible extraer explicaciones y predicciones que estén de acuerdo con las concepciones científicamente compartidas.

Los modelos mentales pueden ser completamente analógicos o parcialmente analógicos y parcialmente proposicionales. En el primer caso, los alumnos tendrían una comprensión más cualitativa, principalmente con la utilización de imágenes mentales, que en general se expresa en la utilización de dibujos o movimientos corporales en sus explicaciones (Greca y Moreira, 1996). En el otro caso, el modelo se caracterizará por el manejo verbal de definiciones y relaciones matemáticas. Es importante destacar aquí que, aunque el alumno «sepa» definiciones o fórmulas, no significa necesariamente que haya construido un modelo; o sea, puede no ser capaz de interpretarlas a la luz de un modelo. A partir de esto, definimos que los alumnos trabajan solamente con proposiciones -o solamente con representaciones proposicionalescuando no consiguen explicar la estructura conceptual de una teoría y los fenómenos vinculados a ella. Comprender la estructura matemática de una teoría -o tener un modelo de su estructura matemática- no es condición suficiente para tener un modelo físico de la teoría; o sea, para comprender la física contenida en esas formulaciones.

Por otro lado, cuando se presenta a los alumnos proposiciones-definiciones, aseveraciones, leyes-, es posible que sean interpretadas a la luz de algún modelo que posean del mundo, que no es necesariamente el compartido por la comunidad científica. Esas proposiciones serían evaluadas como verdaderas o falsas, de acuerdo con la posibilidad de que puedan ser inferidas o no del modelo. Es probable que las «guarden» de manera inconexa, recordándolas sólo como una serie de definiciones y leyes que, apenas pasado el período escolar (o incluso antes), serán olvidadas, dado que los alumnos saben que es eso lo que les será pedido para evaluar su desempeño en la disciplina ${ }^{2}$.

La enseñanza en general, y en particular la de física, no favorece la construcción de modelos. Si revisamos los libros de texto, es posible observar que las teorías aparecen como estructuras acabadas, presentando los fenómenos, leyes y sus expresiones matemáticas de acuerdo con rigurosos criterios lógicos deductivos. Aunque sea evidente que, cuando una teoría científica es presentada, reconstruida racionalmente, responde a estos criterios, eso no significa que la construcción y comprensión por parte del aprendiz sean posibles por medio de estos criterios. Presentar a los estudiantes una serie de postulados y a partir de ahí «inferir» la teoría-como si fuese una rama de las matemáticas- no significa que éstos comprenderán los fenómenos que esa teoría explica.
Tomemos, por ejemplo, el concepto de campo electromagnético, con el cual trabajamos en esta investigación. Si revisamos los libros de texto sobre electromagnetismo, tanto los introductorios como los más avanzados (Halliday y Resnick, 1993; Corson, 1962; Alonso Finn, 1972; Jackson, 1983; Panofsky, 1962), podemos verificar que el concepto de campo juntamente con el de flujo son presentados matemáticamente, formalizando luego (en función de ellos) los fenómenos que serán el sustrato de las ecuaciones de campo. Posteriormente, es presentada la aportación de Maxwell, partiendo de ese sistema de ecuaciones fundamentales para circuitos cerrados, y la ecuación de continuidad de la carga. Su problema será la necesidad de reconciliar esas ecuaciones para el caso de circuitos abiertos. Se dice también que, por cuestiones de consistencia formal, Maxwell agregó un término a la ley de Ampère para representar la contribución de la polarización electrostática a la corriente.

Sin embargo, leyendo al propio Maxwell, podemos ver que las consideraciones de consistencia formal no tuvieron un papel importante en sus construcciones iniciales; deliberadamente no estudió «la matemática de los alemanes», como él expresa, antes de entender las explicaciones de los fenómenos dados por Faraday, y verificó que «muchos de los métodos más fértiles de investigación descubiertos por los matemáticos podrían ser mejor expresados en ideas de Faraday» (Maxwell, 1873), quien no escribió ni una sola fórmula. La elaboración del concepto cuantitativo de campo fue el resultado de la manipulación y del razonamiento sobre una serie de modelos abstractos sucesivos ${ }^{3}$ (Nersessian, 1992). En su Tratado de electricidad y magnetismo (1873), Maxwell se detiene repetidamente en las consideraciones cualitativas del concepto de campo y en la explicación de los métodos que utilizó para concebir los fenómenos antes de pasar a su formalización.

Si los propios científicos, cuando quieren comunicar a sus colegas un nuevo concepto, sienten la necesidad de proponerles analogías físicas que les den sentido y extensos comentarios de cómo comprender el método y las representaciones utilizadas, nos correspondería preguntar por qué creemos que los alumnos pueden aprender algún nuevo concepto solamente a través de consideraciones de consistencia formal.

\section{METODOLOGÍA}

El objetivo de nuestra investigación fue intentar detectar el tipo de representación mental -representaciones proposicionales, imágenes, modelos mentales-que los alumnos utilizaban al resolver problemas y cuestiones teóricas sobre el concepto de campo electromagnético. Asumimos que la estructura de sus representaciones internas se reflejaba de alguna forma en sus representaciones externas. Los estudios en psicología parten del presupuesto de que la forma en que las personas piensan se refleja en el uso del lenguaje, por lo que nosotros consideramos que lo que los alumnos escriban, dibujen y digan nos dará indicios de la forma en que razonaban. 
Las investigaciones realizadas se desarrollaron durante los dos semestres lectivos de 1994. En el primer semestre realizamos un estudio piloto con 31 alumnos de Física General II para Ingeniería, bajo la modalidad del método Keller (Greca y Moreira, 1996). En el segundo semestre, llevamos a cabo el segundo estudio con 25 alumnos que cursaban de manera tradicional, también la Física General II. En ambas ocasiones los alumnos verían por primera vez el concepto de campo electromagnético. En este caso, nuestros objetivos fueron mejorar la categorización del estudio piloto y determinar las características presentadas por los alumnos en las diferentes actividades desarrolladas durante el curso, de acuerdo con el tipo de representación mental utilizada. La metodología y resultados de este segundo estudio son los que presentamos aquí.

Dada la experiencia del estudio piloto consideramos que era necesaria una fuerte interacción con los alumnos. Las clases fueron planificadas para tener un máximo de 30' a 40' de sesión expositiva y durante el tiempo restante (de 65' a 80') los alumnos trabajaban en pequeños grupos sobre trabajos prácticos que incluían, por un lado, preguntas, problemas cualitativos o cuestiones teóricas -cuyo objetivo era que reflexionasen y profundizasen los asuntos tratados en la primera parte de la clase- y, por el otro, problemas de tipo tradicional. [Cuando hablamos de problemas de tipo tradicional hacemos referencia al tipo de los que aparecen como ejercitación básica en los libros de texto (Halliday y Resnick, 1993; Tipler, 1984)]. Para desarrollar estos trabajos prácticos podían discutir entre ellos, consultar al profesor y utilizar el libro de texto. Las hojas conteniendo las respuestas a estos trabajos eran entregadas al final de cada sesión. Durante el curso fueron realizadas tres pruebas formales, que incluían cinco preguntas conceptuales, conteniendo explicación de fenómenos, y cinco problemas tradicionales. En la clase siguiente a cada prueba, los alumnos confeccionaban un mapa conceptual de los conceptos incluidos en la parte de la asignatura que había sido evaluada. Al final del curso, cada uno de los estudiantes fue entrevistado informalmente, durante 10', a 15', acerca de «lo que había aprendido en el curso».

Este tipo de enfoque nos permitió observar diariamente la evolución y rendimiento de los alumnos. Los datos recogidos -anotaciones de campo, trabajos prácticos, evaluaciones y mapas conceptuales- fueron analizados a partir de dos perspectivas: una cualitativa, para determinar el nivel representacional de los alumnos y construir una categorización; y la otra, cuantitativa, para determinar la existencia o no de correlación entre el nivel representacional y el rendimiento escolar. Presentamos aquí el resultado del primero de los dos análisis.

\section{RESULTADOS}

Del análisis cualitativo del material disponible de los alumnos resultaron seis categorías.
Las categorías $0(n=3), 1(n=3)$ y $2(n=3)$ corresponden a los alumnos que no formaron un modelo físico del concepto de campo electromagnético. Trabajan exclusivamente con representaciones proposicionales no integradas en un modelo o, como en el caso de la categoría 2 , con un modelo matemático que les permitía ser muy buenos «resolvedores» de problemas. La diferenciación entre ellos refleja el grado de articulación alcanzada en la utilización de esas representaciones, la manera en cómo encaran los problemas -desde el ensayo y error hasta el desarrollo de algoritmos de resolución y su habilidad en la construcción de mapas conceptuales.

Las categorías $3(n=7), 4(n=7)$ y $5(n=2)$ designan los alumnos que desarrollaron algún tipo de modelo mental físico para el concepto de campo electromagnético. Consideramos la categoría 3 como de transición: en ella fueron incluidos los alumnos que desarrollaron modelos, no siempre correctos, de ciertos conceptos como el de campo eléctrico, pero no alcanzaron a construir uno que incluyese el de campo electromagnético. Los alumnos que los construyeron están en las categorías 4 y 5 .

En la tabla I se encuentran resumidas las características más salientes de cada categoría.

A continuación presentamos respuestas a algunas cuestiones teóricas y algunos mapas conceptuales para ejemplificar y comparar algunos rasgos de las categorías. (Un análisis más detallado aparece en Greca, 1995, anexo).

En la figura 1, podemos distinguir entre el uso de representaciones proposicionales aisladas y las integradas en un modelo. Todas las representaciones en general, son correctas. Sin embargo en la 1.a (categoría 1) aparecen solamente enunciadas las «reglas de los signos» de las cargas y las corrientes, tal como aparecen resumidas en los textos. No se hace referencia a los campos. Más aún, cuando se especifica que «corriente es una cosa y carga es otra», el alumno deja claro que no ha conseguido integrar los conceptos en un modelo; para él, son conceptos aislados. En las respuestas 1.b y 1.c (categoría 3), los conceptos comienzan a relacionarse, aunque esta relación sea débil y las explicaciones todavía bastante próximas a las del libro. Esta categoría fue justamente considerada de transición, pues tanto las proposiciones como las imágenes aparecen, a veces, aisladas y otras, integradas (como en la $1 . b, F$ y $E$ ), evidenciando la construcción de modelos de ciertos conceptos. Cuando esto ocurre, sus explicaciones se alejan de la repetición textual. Dos de las características que detectamos a lo largo del curso en la categoría 3 son ejemplificadas en estas respuestas: 1) Tienden a insistir en la utilización de las reglas de construcción de las líneas de campo; estas reglas son especificadas continuamente, tanto en sus respuestas orales (a veces mediante el uso de las manos) como en las escritas. Parece que las imágenes mentales que forman de las líneas de campo tengan que estar amarradas a representaciones de entidades reales. 2) En esta categoría, los alumnos comienzan a utilizar dibujos. 


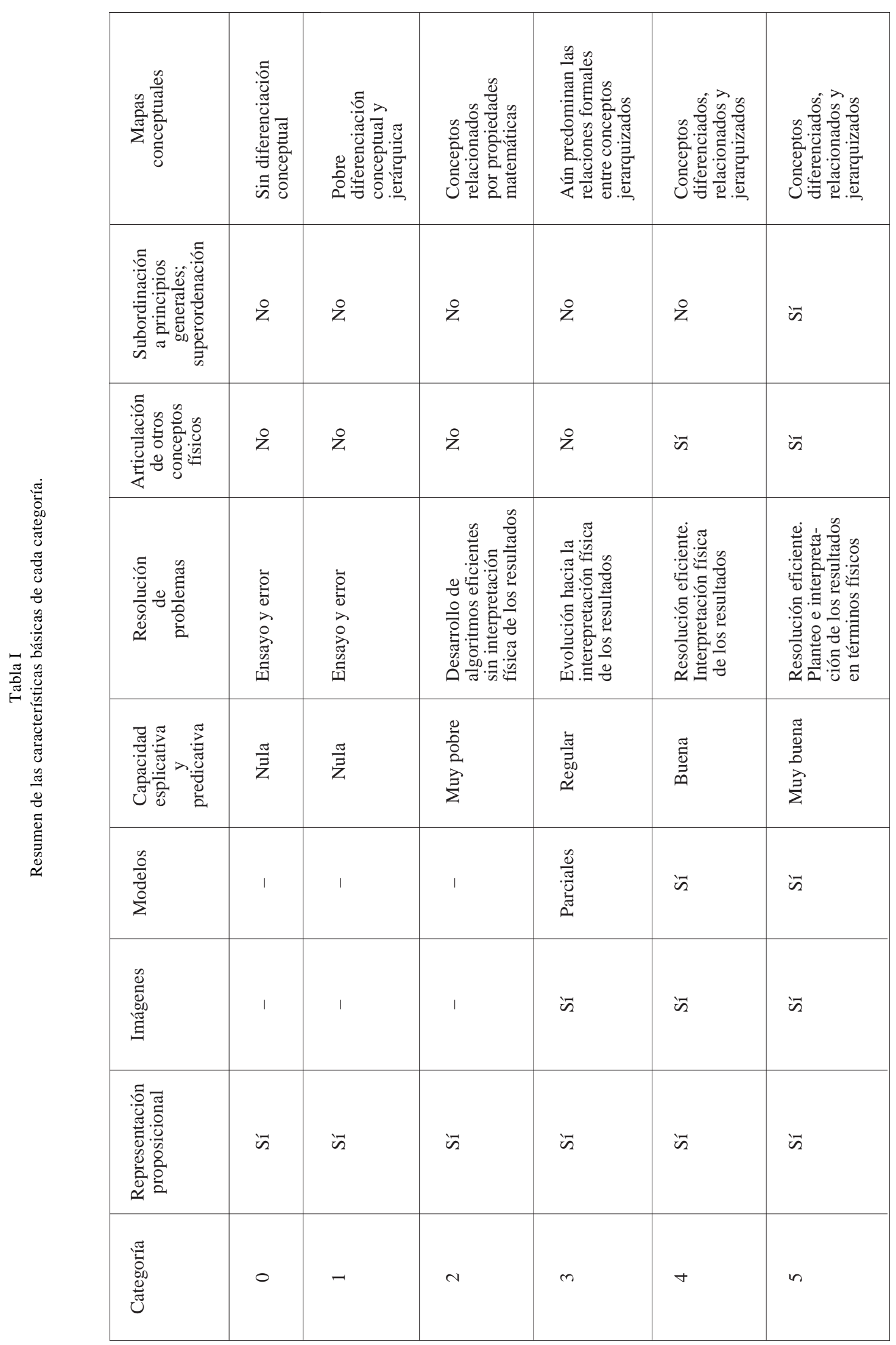


Los alumnos de la categoría 4, cuyas respuestas aparecen en 1.d y 1.e, forman un modelo físico de campo eletromagnético que les permite interpretar los fenómenos de manera integrada. Los modelos subyacentes no son iguales para todos. Por ejemplo, en la 1.d, la explicación es en términos más formales y referida a las leyes que gobiernan cada tipo de fenómeno (modelo básicamente proposicional) en tanto que, en la 1.e, la explicación se centra en las relaciones entre los campos, además de la utilización de diseños

Las respuestas 2.a y 2.b corresponden a alumnos de las categorías 0 y 4 respectivamente. Con ellas queremos ejemplificar la diferencia de función que ocupan los diseños en las respuestas. En el primer caso, no tienen ninguna función: es repetición de una figura del texto, que ni siquiera está relacionada con la respuesta que da al problema. (Una característica de la categoría 0 es que los alumnos presentaron serias dificultades en la resolución de problemas, excepto en aquéllos en que debían aplicar una sola fórmula). En el segundo caso, los diseños ganan capacidad explicativa y responden a imágenes relacionadas con un modelo. Los diseños son, en varios de los alumnos de esta categoría, reflejo de las visualizaciones que manifestaron precisar para «imaginar lo que ocurría» antes de resolver un problema. Este caso en particular es de un alumno cuyo modelo era básicamente analógico.

El carácter «ornamental» de algunos de los diseños (o sea, diseños que no reflejan la utilización de imágenes mentales como herramientas para el razonamiento) se refleja también en la figura 3.a (categoría 2). El alumno repite de memoria el gráfico que aparece en el texto. Las líneas de campo magnético no tienen utilidad, ni siquiera consiguen saber hacia adónde apuntan. Los alumnos de la categoría 2 trabajaron desde el punto de vista proposicional de manera muy eficiente, sobre todo en la articulación de la estructura matemática del electromagnetismo. Sin embargo, su objetivo nunca fue entender físicamente lo que ocurría. Su interés nacía y moría en saber cómo utilizar las ecuaciones. El otro caso, figura 3.b, revela otra característica de la categoría 4: las ecuaciones comienzan a tener un papel secundario, en el sentido en que no son usadas como principio y fin de la comprensión. Están asociadas a los conceptos que representan, pero son éstos el objeto cuya comprensión es necesaria. Para los alumnos de las categorías 4 y 5 , comprender implicaba poder explicar los fenómenos en términos cualitativos.

La figura 4.a corresponde a un alumno de la categoría 2. Como mencionamos antes, reveló sobrestimar el valor de las expresiones matemáticas en la explicación de los fenómenos. Cuando esto no era posible, repetía lo que aparecía en sus anotaciones, como vimos en la figura 3.a. En general sus explicaciones del campo electromagnético son confusas, tal como muestra la figura 4.a. En la 4.b. aparece parte de la respuesta de un alumno de la categoría 4 a la misma cuestión. Nuevamente, las explicaciones de las ecuaciones remiten a conceptos físicos (líneas de campo, monopolo magnético). Ya la figura 4.c (categoría 5) muestra porque diferenciamos entre los alumnos de las categorías 4 y 5 . Si bien consideramos que ambos grupos habían construido modelos físicos del concepto de campo, los de la categoría 5 formaron modelos más «abarcativos», integrando el concepto de campo electromagnético con leyes fundamentales de la física, en este caso la conservación de la energía.

La figura 5 corresponde a distintos mapas conceptuales (sobre electricidad, magnetismo y electromagnetismo) de alumnos de diferentes categorías. El 5.a (categoría 0) refleja un aprendizaje memorístico y superficial, resultante en un diagrama de títulos de los capítulos del libro, no consiguiendo siquiera identificar cuáles fueron los conceptos enseñados. En el 5.b (categoría 1), no hay diferenciación entre conceptos y leyes. Las relaciones, cuando aparecen, reúnen un par de conceptos a través de definiciones del libro o fórmulas (representaciones proposicionales aisladas). En el 5.c (categoría 2) aparece nuevamente la característica más destacada de los alumnos de esta categoría: las relaciones entre los conceptos son fórmulas. Tampoco existe una jerarquía de conceptos (no hay modelo físico).

En el 5.d (categoría 3) comienza a evidenciarse una cierta diferenciación conceptual y jerárquica, esto evidenciaría que, de alguna forma, los conceptos comienzan a estructurarse en sus cabezas, no son sólo definciones aisladas. Algunas de las relaciones no son correctas (como señalamos antes, los minimodelos de esta categoría no eran siempre correctos desde el punto de vista científico). Los mapas 5.e y 5.f reflejan las diferencias entre los modelos construidos por los alumnos de la categoría 4: los campos son pensados o imaginados de manera diferente. En el caso de la figura 5.f, parece estar más relacionado con los generadores de los campos: cargas, corrientes, etc. El mapa 5.g corresponde a un alumno de la categoría 5 . El carácter simétrico de los fenómenos eléctricos y magnéticos, su relación dinámica en el campo electromagnético y su resultado (las ondas electromagnéticas) aparecen claramente. Fue por esta causa que consideramos que los alumnos de esta categoría construyeron modelos que corresponderían a los modelos usados por un especialista en este nivel.

(A excepción de los alumnos de la categoría 0 , el resto de los alumnos aprobó el curso. De hecho, en el análisis cuantitativo, las correlaciones entre la formación de modelos y rendimiento en la resolución de problemas no era alta. Dado que en los problemas tradicionales, que es el tipo de problemas exigidos para la aprobación del curso, no es relevante la comprensión física sino que se evalúa muchas veces la pericia matemática, aquellos alumnos que trabajan sólo proposicionalmente consiguen desarrollar algoritmos eficientes de resolución. Probablemente ésa sea la visión que tengan del aprendizaje de física).

\section{IMPLICACIONES PARA LA INVESTIGACIÓN Y LA ENSENANZA}

En este punto, analizaremos algunas de las posibles consecuencias del enfoque que adoptamos, que conside- 
ramos interesantes tanto para fines instruccionales como para futuros trabajos de investigación. Queremos, a su vez, enfatizar que éste es solamente un estudio preliminar y que se debe ser cauteloso en la extrapolación de conclusiones. Así, no es nuestra intención prescribir métodos o técnicas, lo que sería imposible dado el tipo de preguntas que nos propusimos, sino plantear algunos elementos de análisis que pueden servir para profesores e investigadores.

El aspecto más importante de los resultados encontrados en esta investigación se refiere al hecho de que sugieren que representaciones mentales, tales como las definidas por Johnson-Laird, son importantes en el momento de hacer y aprender física.

$\mathrm{Si}$ es cierto que las personas razonan a partir de esos modelos que describimos, sería posible reinterpretar el movimiento de las concepciones alternativas desde este punto de vista. Las ideas previas de los alumnos no son concepciones aisladas, ilógicas o incoherentes, sino que estarían organizadas en estos modelos de trabajo, lo que les daría la estabilidad que es comprobada por las investigaciones en el área. Los alumnos construyen naturalmente modelos causales simples de sistemas físicos que les sirven para entender el mundo que los rodea. Ésa es la matriz de los modelos intuitivos. Esos modelos son generados por la percepción, por el ambiente social o por el contexto escolar. Parte de la fuerte resistencia al cambio de esos modelos puede resultar de que son eficientes para entender el mundo antes de entrar en la escuela, y lo continúan siendo fuera de las aulas de ciencia. «Cuando no se aplican fuerzas -vinculadas al esfuerzo o a combustibles-, las cosas no se mueven», es parte de un modelo muy eficiente para un mundo con rozamiento.

Interpretar un enunciado científicamente compartido significa verlo a la luz de un modelo del mundo, donde ese enunciado tenga sentido; o sea, que pueda ser evaluado como verdadero. Eso sólo será posible si fuese aprehendida la estructura conceptual científica en la que ese enunciado se justifica. Ese proceso será más que una simple reordenación de conceptos existentes o adición de hechos a la estructura conceptual anterior, o eliminación «iluminada» de un modelo por otro: implica la construcción de un nuevo modelo de trabajo que debe estar en parte anclado en los modelos antiguos de los alumnos. Los modelos intuitivos pueden pasar a ser, en el mejor de los casos, uno de los hipotéticos modelos de mundo que pueden ser construidos para interpretar la realidad, y el científicamente compartido será otro de ellos. Existirá, entonces, una discriminación de modelos, una forma de adaptar cada uno de acuerdo con el contexto en que será utilizado (Moreira, 1993).

Una línea interesante de investigación se refiere a cómo ese proceso de génesis y evolución de los modelos mentales es influenciado por la escolaridad. En la introducción indicamos que, si una proposición no era verdadera de acuerdo con un modelo de mundo, sería considerada falsa y descartada -o mantenida en mente en cuanto fuese necesaria para aprobar la disciplina. Ahora bien, ¿que ocurriría si fuese presentada a los alumnos una proposición nueva, que no pudiese ser considerada como falsa en su modelo de mundo (suponiendo que el alumno tenga un modelo en donde esa proposición pueda encajar)? ¿Cómo se modificaría ese modelo con la perturbación? Si no consigue construir un nuevo modelo, ¿cuál será la forma de ese híbrido?

Uno de los posibles ejemplos de esta cuestión es el caso de la fuerza de la gravedad. En las investigaciones sobre concepciones alternativas de los alumnos sobre la caída libre, aparece con frecuencia que los alumnos indican la existencia de una fuerza que apunta hacia abajo, asociándola con la gravitacional. Cuando se les pide que diseñen el diagrama de fuerzas en el caso de una piedra lanzada hacia arriba, se observa que dibujan dos vectores, uno en la dirección del movimiento, para arriba, y otro apuntado hacia abajo. Si tomamos ambos diseños conjuntamente, no conseguiríamos clasificarlo como un modelo intuitivo coherente que corresponda a la relación fuerza proporcional a velocidad. Sin embargo, podríamos analizarlo de esta forma. El alumno tiene un modelo en que la velocidad tiene el sentido de la fuerza, tanto en el primer caso, apuntando hacia abajo -todo los cuerpos caen-, como en el segundo, apuntando hacia arriba -fuerza impresa por él a la piedra. En la escuela aprenden que en ambos casos la única fuerza que actúa es la gravitacional, que apunta hacia abajo. En el primer caso puede asociar su idea automáticamente con el concepto de fuerza gravitacional y en el segundo, como la piedra lanzada hacia arriba luego caerá, la existencia de la fuerza gravitacional no entrará frontalmente en conflicto con su modelo. La perturbación escolar provocaría este tipo de respuesta híbrida, que no es ciertamente lo que el profesor quería que aprendiese.

En un interesante trabajo de estudio de caso sobre las ideas de los alumnos, Kuiper (1994), después de realizar un análisis detallado de tests aplicados a alumnos en cuatro áreas diferentes de problemas sobre el concepto de fuerza (cuerpos en reposo, en movimiento, lanzados y colisiones), encontró que la mayoría de los alumnos utilizan el mismo tipo de ideas sobre fuerzas en una misma área pero que, en áreas distintas, usan otras esencialmente diferentes -y en conflicto con las concepciones científicas. Solamente del grupo de estudiantes más jovenes, o sea con menor grado de escolaridad, podía decirse que tenían un framework intuitivo alternativo y coherente que correspondía la relación fuerza proporcional a velocidad. Concluye que los alumnos en general no tienen un framework para fuerza, sino un conjunto vago de ideas incoherentes. Si miramos esta investigación a la luz de lo que venimos diciendo hasta aquí, podríamos decir que lo que aparece en los alumnos más pequeños es que aún mantienen su modelo intuitivo, en cuanto que aquéllos que recibieron instrucción en ciencias por algún tiempo desarrollan algunos modelos híbridos, y otros sólo «repiten» proposiciones, retomando sus modelos intuitivos cuando están frente a situaciones no idénticas a las cuales aprendieran las proposiciones anteriores. Las implicaciones didácticas resultantes de uno y otro análisis son diferentes. 
De acuerdo con el primer análisis, será necesario ayudar a los alumnos a hacer su razonamiento conceptual más coherente. En los niños menores, se debería concentrar en modificar cada una de las ideas incorrectas y, en los más avanzados [los que podrían realizar inferencias operacionales formales tales como las definidas por Piaget e Inhelder (1956)], se debería dar mayor atención a la estructuración y formalización de varias ideas en el «lógico y coherente framework científico» (Kuiper, 1994, p. 290). Sin embargo, corregir ideas aisladas no llevará a la construcción de un nuevo modelo, ni consideraciones sobre la consistencia lógica y la coherencia de las ideas científicas las tornarán mejores que los modelos de trabajo de los propios alumnos.

Es preciso que el alumno comprenda las teorías científicas como un tipo de modelo diferente al suyo, como otra forma de ver el mundo, con algunas reglas de juego particulares -como la posibilidad de definir operacionalmente y cuantificar sus conceptos. Pero, en principio, deberá aprender el marco conceptual de la teoría, generando una representación interna del mismo. Se podría ayudar a los alumnos a construir esos modelos, enseñándoles las formas con las cuales los científicos generan sus propias representaciones (a este respecto, puede

\section{REFERENCIAS BIBLIOGRÁFICAS}

ALONSO, M. y FINN, E. (1972). Física: Um curso universitário. Vol. II. Sao Paulo: Editora Edgard Blucher Ltda.

CORSON, D. y LORRAIN, P. (1962). Introduction to electromagnetic fields and waves. San Francisco: W.H. Freeman and Company.

EISENCK, M. y KEANE, M. (1990). Cognitive psychology: a student's handbook. Londres: Erlbaum.

GRECA, I. (1995). Tipos de representaçoes mentais - modelos, proposições e imagens - utilizadas por estudantes de física geral sobre o conceito de campo eletromagnético. Dissertacão de Mestrado. Porto Alegre: IFUFRGS.

GRECA, I. y MOREIRA, M. A. (1996). Un estudio piloto sobre representaciones mentales, imágenes, proposiciones y modelos mentales respecto al concepto de campo electromagnético en alumnos de física general, estudiantes de postgrado y físicos profesionales. Investigações em Ensino de Ciências, Vol. 1(1).

HALLIDAY, D. y RESNICK, R. (1993). Fundamentos de física 3. Río de Janeiro: Livros Técnicos e Científicos Editora.

JACKSON, J. (1983). Eletrodinâmica clássica. Río de Janeiro: Guanabara Dois.

JOHNSON-LAIRD, P. (1983). Mental models. Cambridge: Cambridge University Press.

JOHNSON-LAIRD, P. (1990). Mental models, en Posner, M. (ed.), Foundations of cognitive science. Cambridge, MA: MIT Press, pp. 469-499. verse el trabajo de Nersessian, 1992). Así el formalismo que ciertamente es indispensable adquirirá sentido, dejando de ser tan sólo fórmulas que caen en el vacío.

\section{NOTAS}

1 Si pensamos que el centro del planteamiento de la psicología cognitiva es la exploración de la analogía entre el computador y la mente, así como el computador, en último análisis, sólo computa a partir de cadenas de unos y ceros, la mente también sólo computaría desde el punto de vista proposicional, o sea, con cadenas de símbolos mentales primitivos. Sin embargo, Johnson-Laird (1983) argumenta que los modelos y las imágenes mentales son especies de lenguajes de programación de alto nivel para la mente, en el sentido de que la liberan de trabajar en el «código de máquina», de la misma forma que lenguajes computacionales más desarrollados facilitan enormemente la tarea del programador.

2 Los modelos mentales son más fáciles de recordar, pues requieren mayor cantidad de procesamiento para ser construidos (Mani y Johnson-Laird, 1982).

3 La función de esas representaciones imaginarias, tanto en Faraday como en Maxwell, sería la de ofrecer un nivel de análisis fructífero entre el fenómeno y las formas matemáticas de representación (Nersessian, 1992).

KUIPER, J. (1994). Students ideas of science concepts: alternative frameworks? International Journal of Science Education, Vol. 16(3), pp. 279-292.

MAXWELL, J. (1954). A treatise on electricity and magnetism. (Unabridge 3rd edition). Nueva York: Dover Publications, INC.

MOREIRA, M. A. (1994). Cambio conceptual: crítica a modelos actuales y una propuesta a la luz de la teoría del aprendizaje significativo. Proceedings of the International Conference «Science and Mathematics Education for the 21st century: Towards Innovatory Approaches». Universidad de Concepción. Chile.

NERSESSIAN, N. ( 1992). How do scientists think? Capturing the dynamics of conceptual change in science, en Cognitive models of Science, Vol. XV. Minnesota Studies in the X Philosophy of Science. Minneapolis: University of Minnesota Press. pp. 3-44.

PANOFSKY, W. y PHILLIPS, M. (1962) . Classical electricity and magnetism. 2a. ed. Massachussetts: Addison-Wesley Publishing Company.

PIAGET, J. y INHELDER, B. (1995). De la logique de l'enfant a la logique de l'adolescent. París: Presses Universitaires de France.

POZO, J. et al. (1994). La solución de problemas. Madrid: Santillana.

TIPLER, P. (1984). Física. Vol. 2 (21a. ed.). Río de Janeiro: Guanabara Dois. 


\section{ANEXO}

\section{Figura 1}

Respuestas a la pregunta: Corrientes del mismo sentido se atraen y de sentido contrario se repelen; cargas estacionarias del mismo signo se repelen y de signos contrarios se atraen. ¿Es esto una paradoja?

1.a: No es una paradoja. Corriente es una cosa y carga es otra. En las corrientes del mismo sentido, las fuerzas apuntan en el mismo sentido y se suman. Ya en las cargas, las fuerzas son opuestas, por eso se repelen.

1.b: No. Primero, carga y corriente no son magnitudes iguales; en la corriente tenemos: corriente $\rightarrow$ cargas en movimiento.
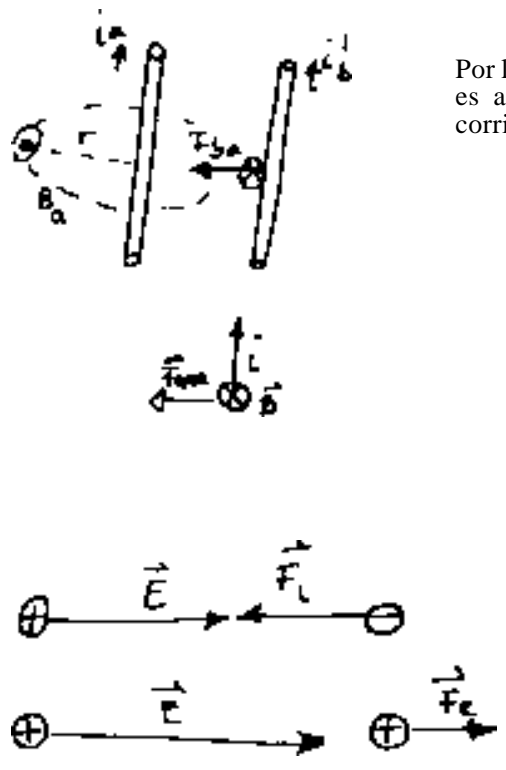

Por la regla de la mano derecha, la fuerza ejercida en $a$ por $b$ es atractiva o repulsiva dependiendo del sentido de las corrientes.

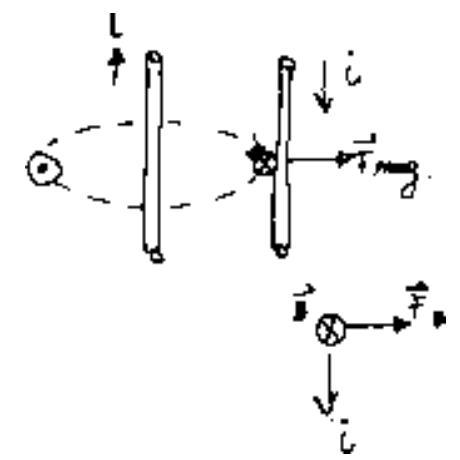

1.c: No, porque las cargas no interfieren en el sistema de campo magnético, sólo indican el sentido; así, en las corrientes aquéllas que tienen el mismo signo se atraen del mismo modo que las cargas de signos contrarios se atraen.

1.d: La paradoja no existe, pues estamos hablando de situaciones diferentes. En el primer caso, la interacción que ocurre entre los conductores es relativa al campo magnético y la consecuente fuerza magnética que proporcionan ese efecto de atracción/repulsión. En el segundo caso es diferente, ya que los elementos del sistema (cargas estacionarias) obedecen los principios de la electrostática y no del magnetismo.

1.e: No, pues en el caso de las cargas en movimiento (corriente) ocurre que éstas producen un campo magnético, hecho que no ocurre en el caso de las cargas estacionarias. Son esos campos magnéticos los responsables de la fuerza que irá a repeler o a atraer la corriente.

En el caso de corrientes iguales, tenemos el siguiente esquema:
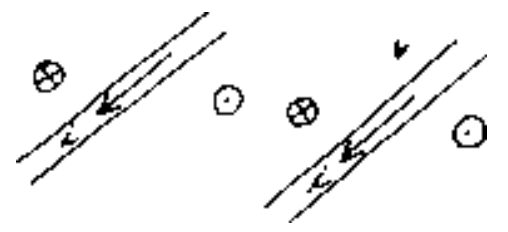

Por otro lado, en el caso de corrientes opuestas, tenemos:

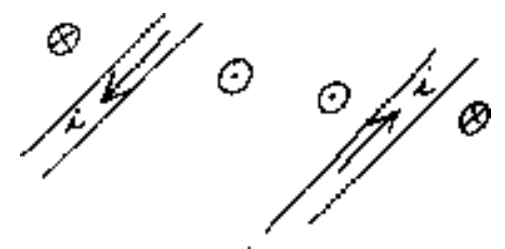

ENSEÑANZA DE LAS CIENCIAS, 19988, 16 (2)
Como en el espacio entre los cables tenemos campos opuestos, éstos van a atraerse, lo que provoca una atracción de las corrientes.

Aquí ocurre lo contrario, pues tenemos campos iguales entre los cables y que, por lo tanto, van a repelerse. 
Figura 2.a

Respuesta a la pregunta: Obtener una expresión para el módulo del campo magnético en el interior de un hilo conductor cilíndrico, con corriente i. Se puede imaginar este plano como una concatenación de infinitos hilos de diámetro tendiendo a cero.

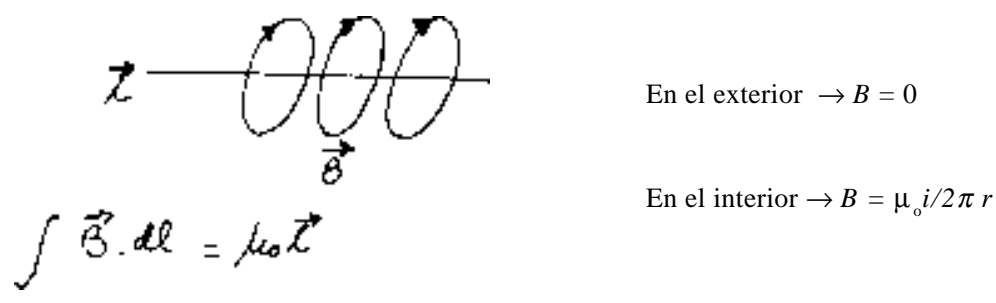

Figura 2.b

Respuesta a la pregunta: Obtener una expresión para el módulo del campo magnético de un plano infinito.

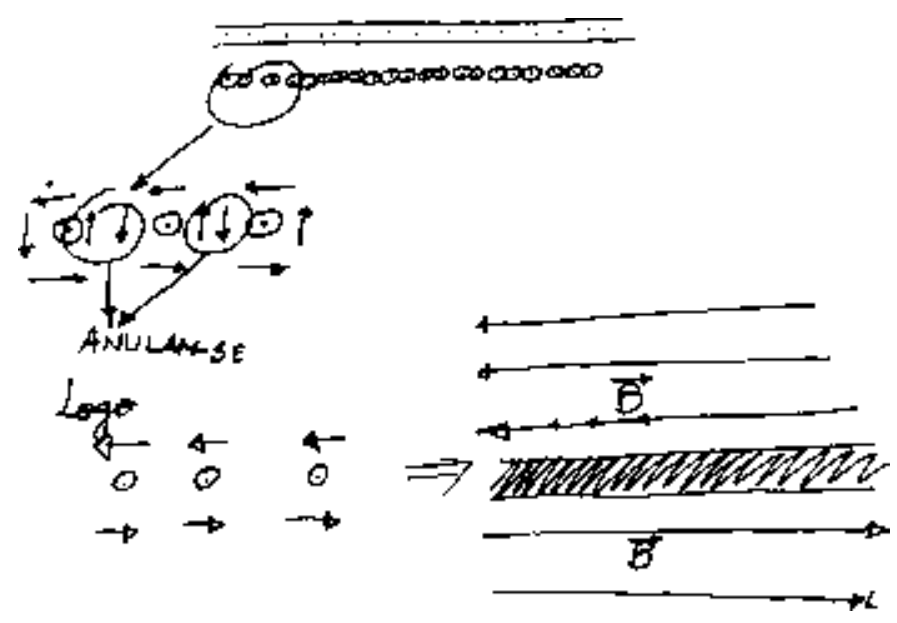

Y como el plano es infinito, no importa la distancia a la que estamos del plano, ya que la intensidad del campo será la misma.

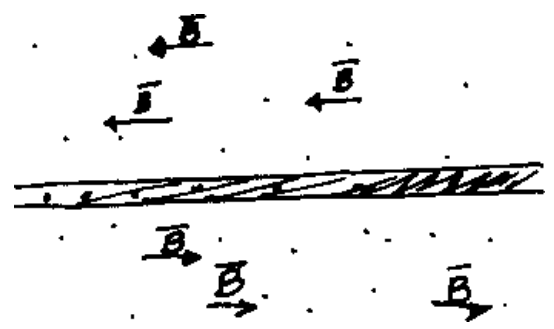


Respuestas a la pregunta: Utilize dibujos, explicaciones o ecuaciones para describir los campos eléctrico y magnético del capacitor y del inductor en un instante cualquier en que ambos estén cargados.

3.a:

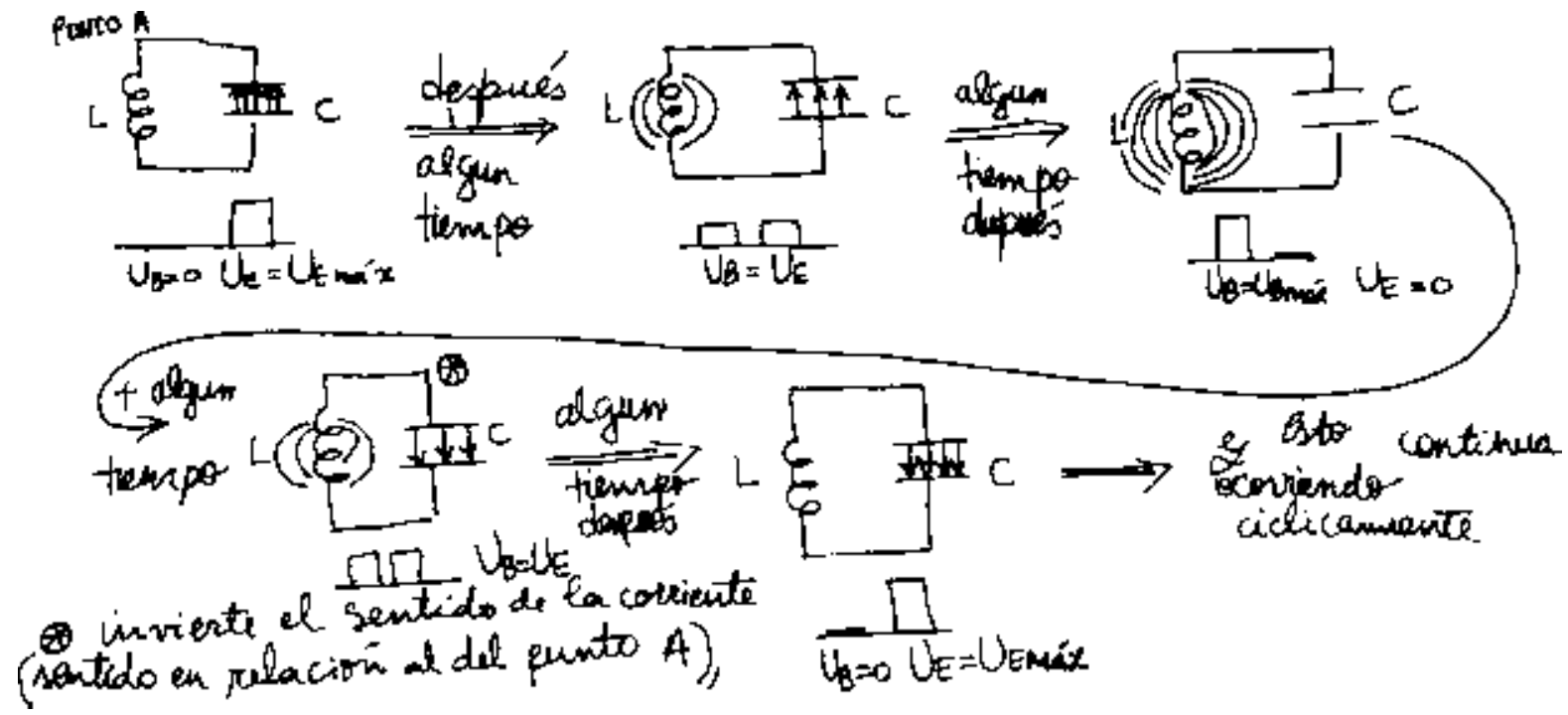

3.b: En un circuito LC oscilante, cuando ambos están «cargados», uno de ellos debe estar cargando y el otro descargando. Si el inductor está cargando, eso significa que el campo magnético está aumentando y, así, el campo eléctrico está disminuyendo. En el caso de que el capacitor esté cargando, ocurre la situación inversa: el campo magnético está disminuyendo al paso que el campo eléctrico está aumentando. Eso ocurre, pues, al cargar, el inductor almacena energía a través del campo magnético, ya que $\mathrm{U}=1 / 2 \mathrm{Li}^{2}$ y $\mathrm{L}=\mathrm{N} \phi \mathrm{B} / \mathrm{i}$, y ocasiona una disminución del campo eléctrico, pues $\oint \mathrm{E} . \mathrm{dl}=\mathrm{d} \phi \mathrm{B} / \mathrm{dt}$. Análogamente, cuando el capacitor carga, la energía es almacenada a través del campo eléctrico, pues $U=1 / 2 \mathrm{q} / \mathrm{c}$ y E.ds $=\mathrm{q} / \varepsilon_{\mathrm{o}}$, ocasionalmente la disminución del campo magnético.

Figura 4

Respuesta a la pregunta: Utilize dibujos o explicaciones para dar una idea de lo que entiende por campo electromagético.

4.a: Un campo eléctrico puede ser producido por una corriente o por una carga eléctrica puntiforme, así como por un campo eléctrico variable. Un flujo magnético variable está relacionado con un campo magnético producido por una corriente.

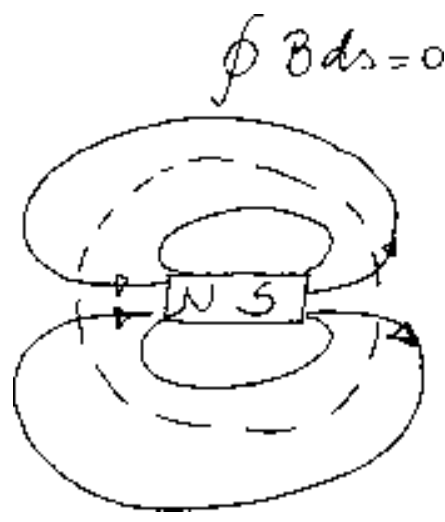

4.b: Ley de Gauss para el magnetismo: Si considerarmos una superficie cerrada, el flujo $\oint$ B.ds será siempre nulo, pues todas las líneas que entran, salen. Distinto de un polo eléctrico, no existe un polo magnético aislado, al que podamos envolver con alguna superfície.

4.c: Los campos eléctricos y magnéticos son un medio de energía almacenada. Si fuera colocada, en una región de campo eléctrico, una carga, por ejemplo, sufrirá una alteración, o sea, una variación de energía cinética. Esta energía viene del campo eléctrico en que está. Un concepto más restringido a la eletrostática dice que el campo eléctrico indica la presencia de una carga, pero no es adecuado para describir fenómenos más complejos como ondas. Los campos eléctricos y magnéticos poseen una relación muy estrecha, pues se sabe que la variación de uno de ellos induce el surgimiento del otro en una dirección perpendicular (ec. de Maxwell). E1 fenómeno de la inducción nos puede llevar a pensar en una sucesión de campos eléctricos y magnéticos propagándose, en determinada dirección por el espacio: esto es una onda electromagnética. 


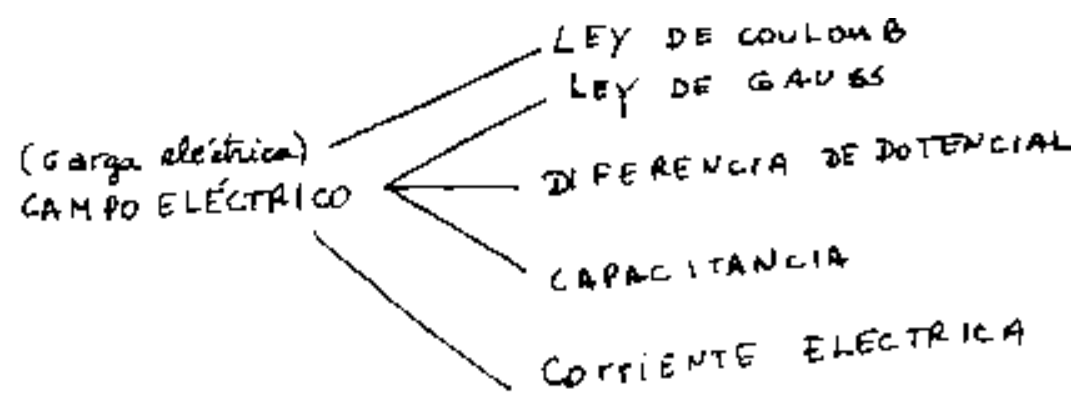

ELECTROMA ONETISMO

(MAGNETISHO) chmo magnético
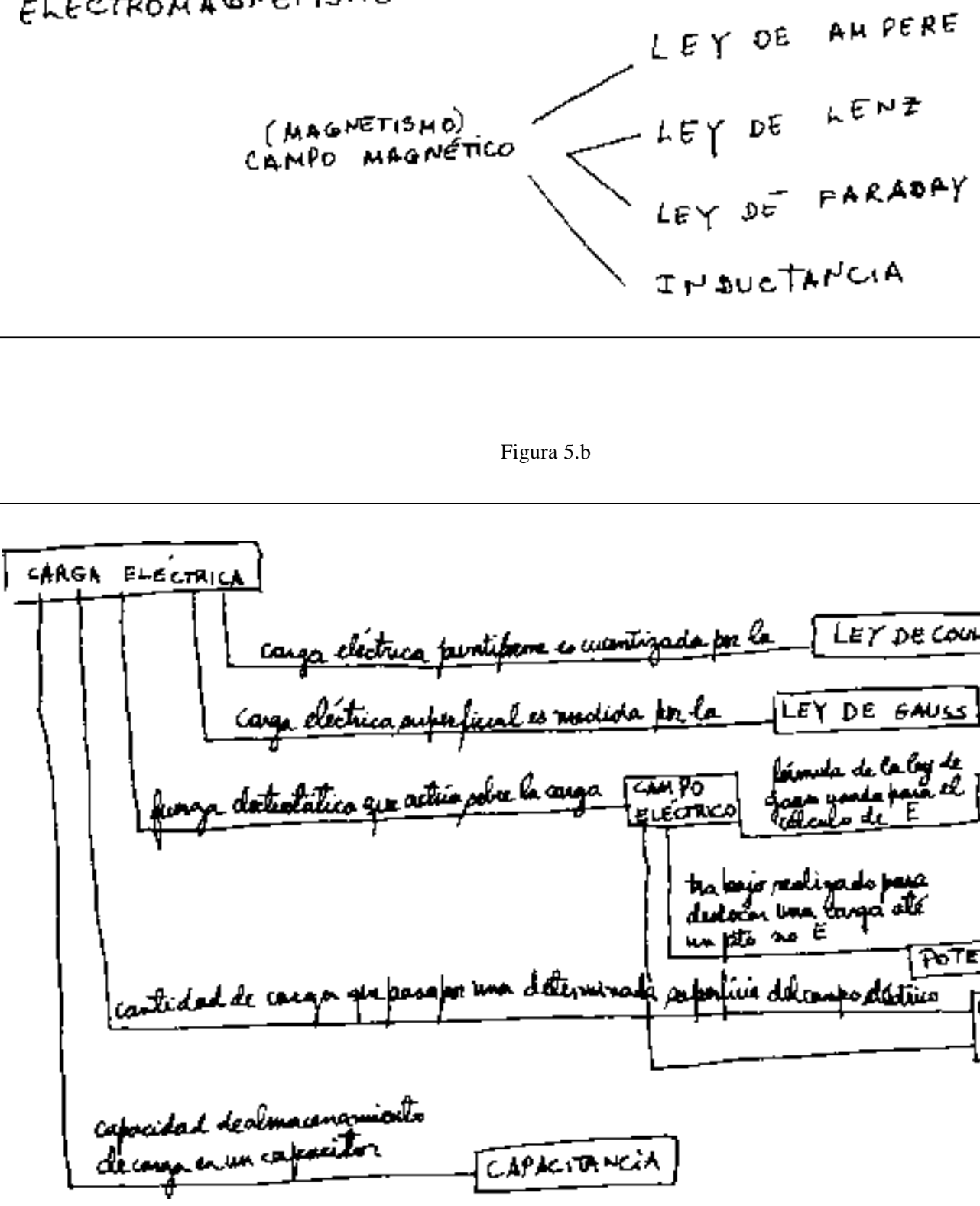


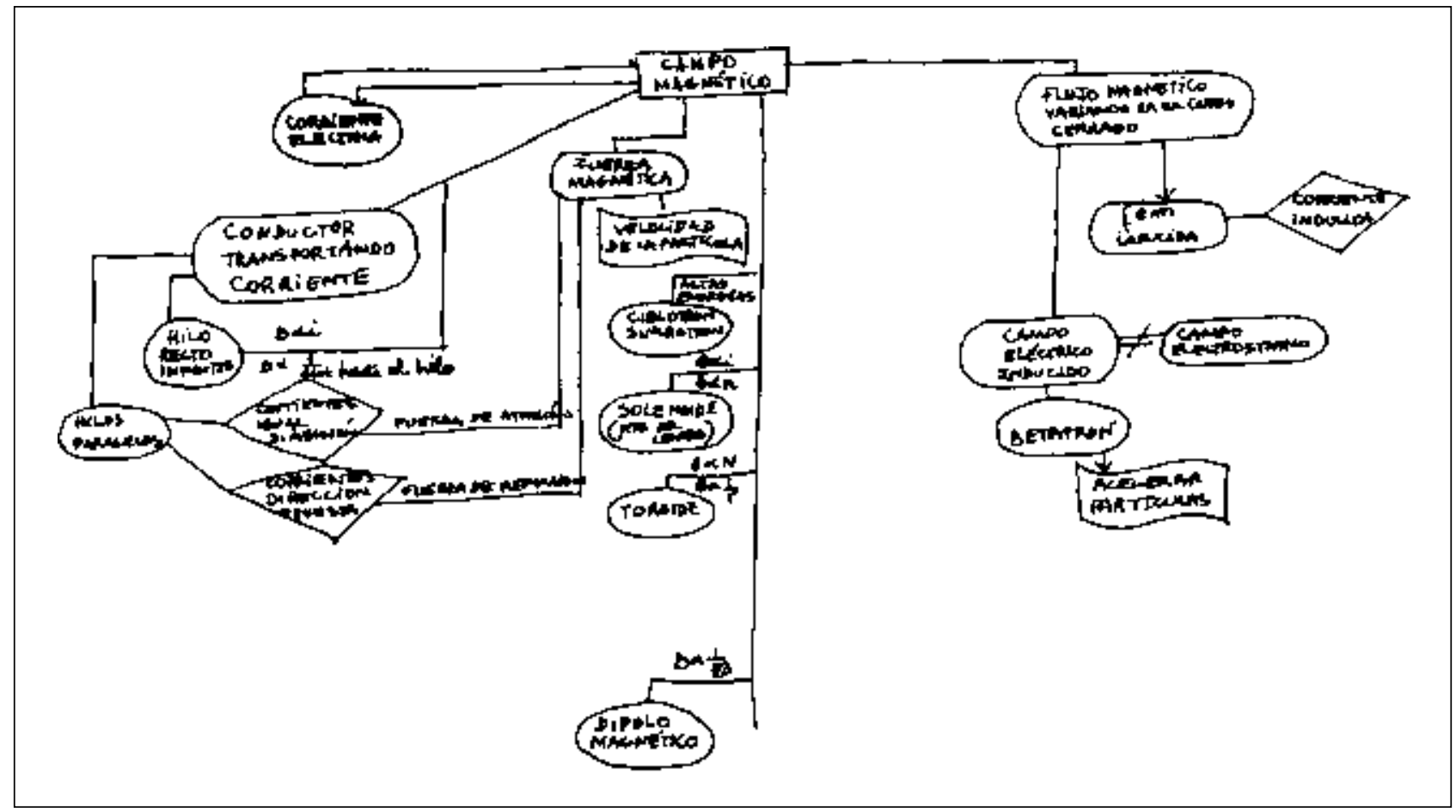

Figura 5.d

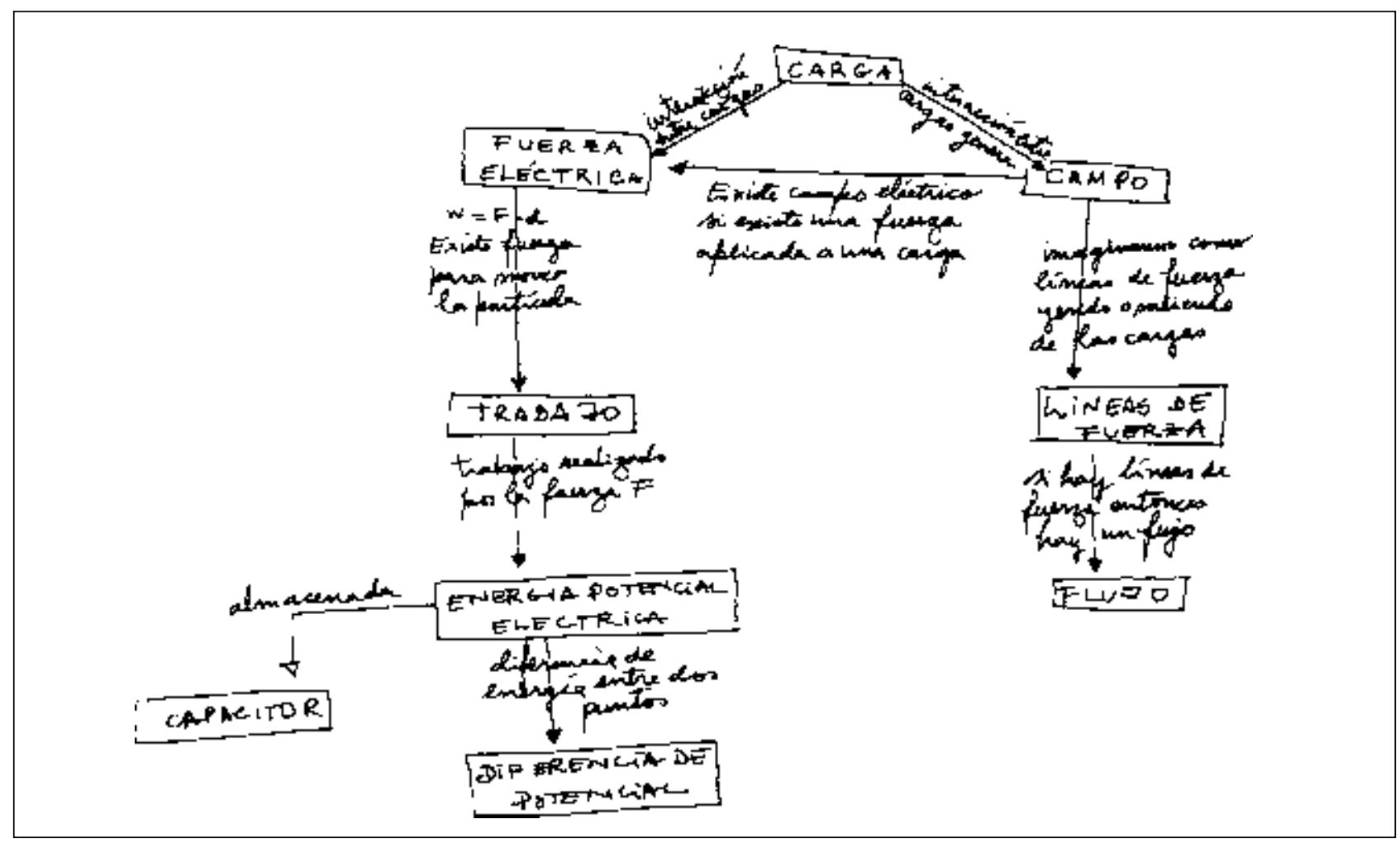




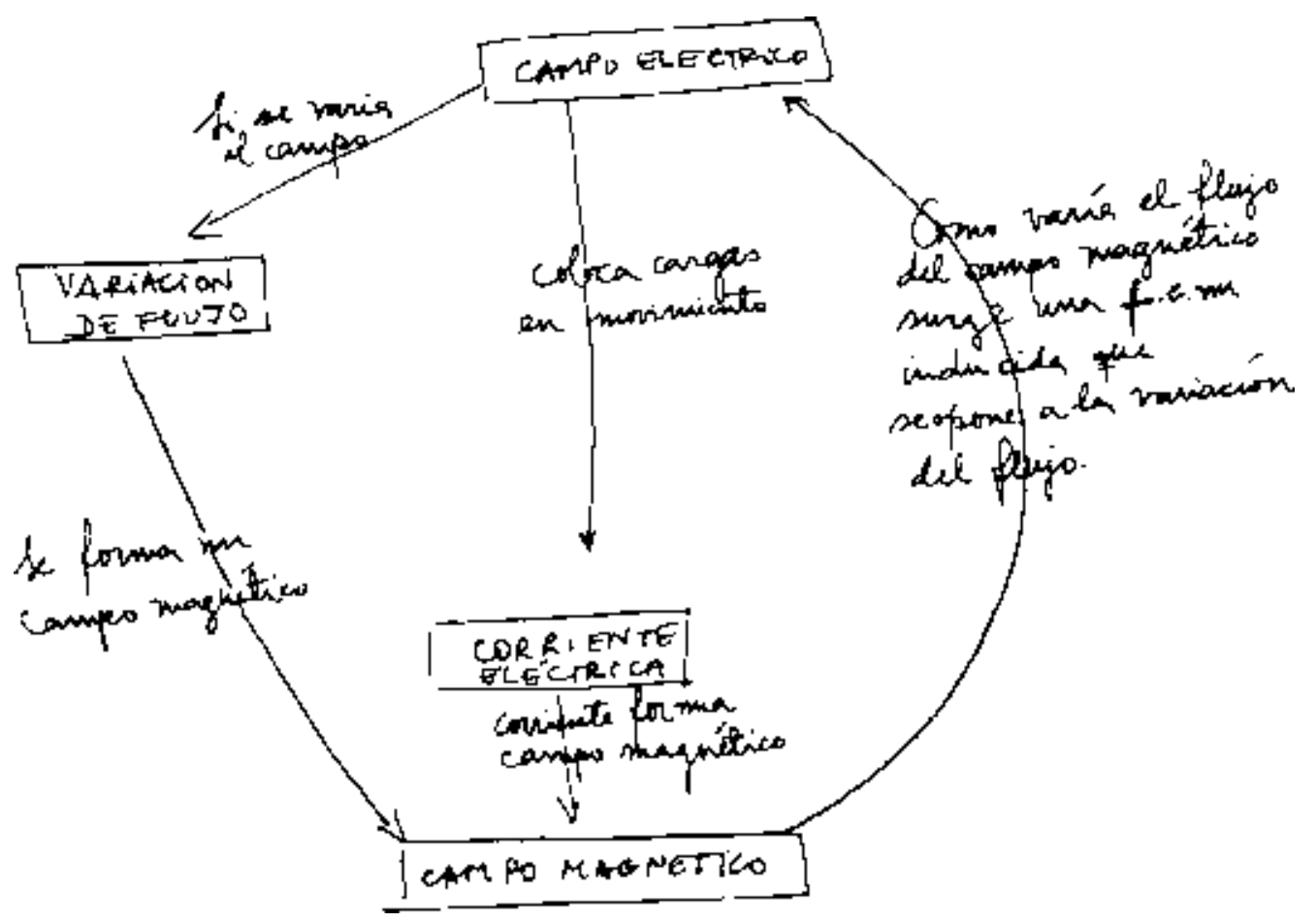

Figura 5.f

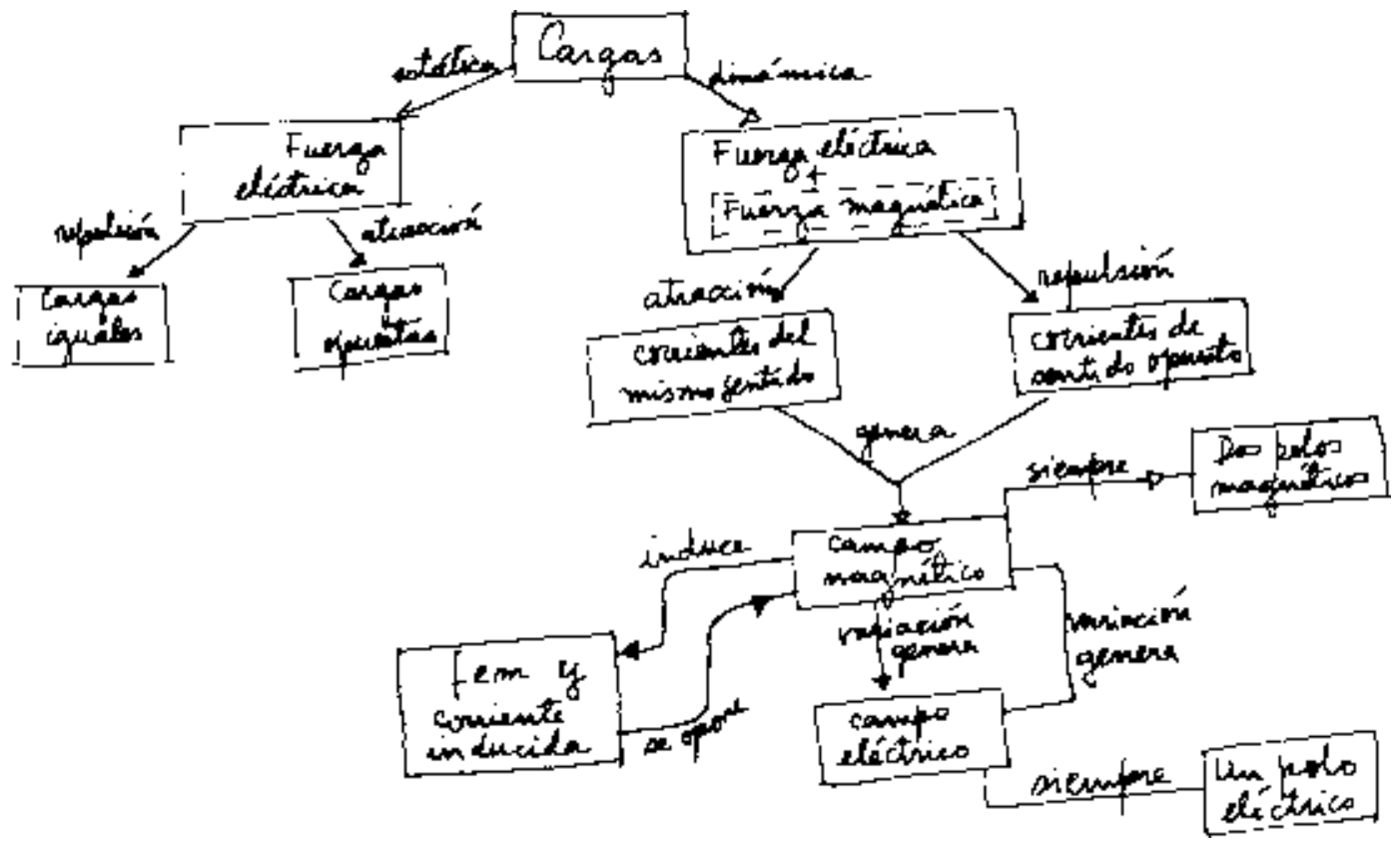




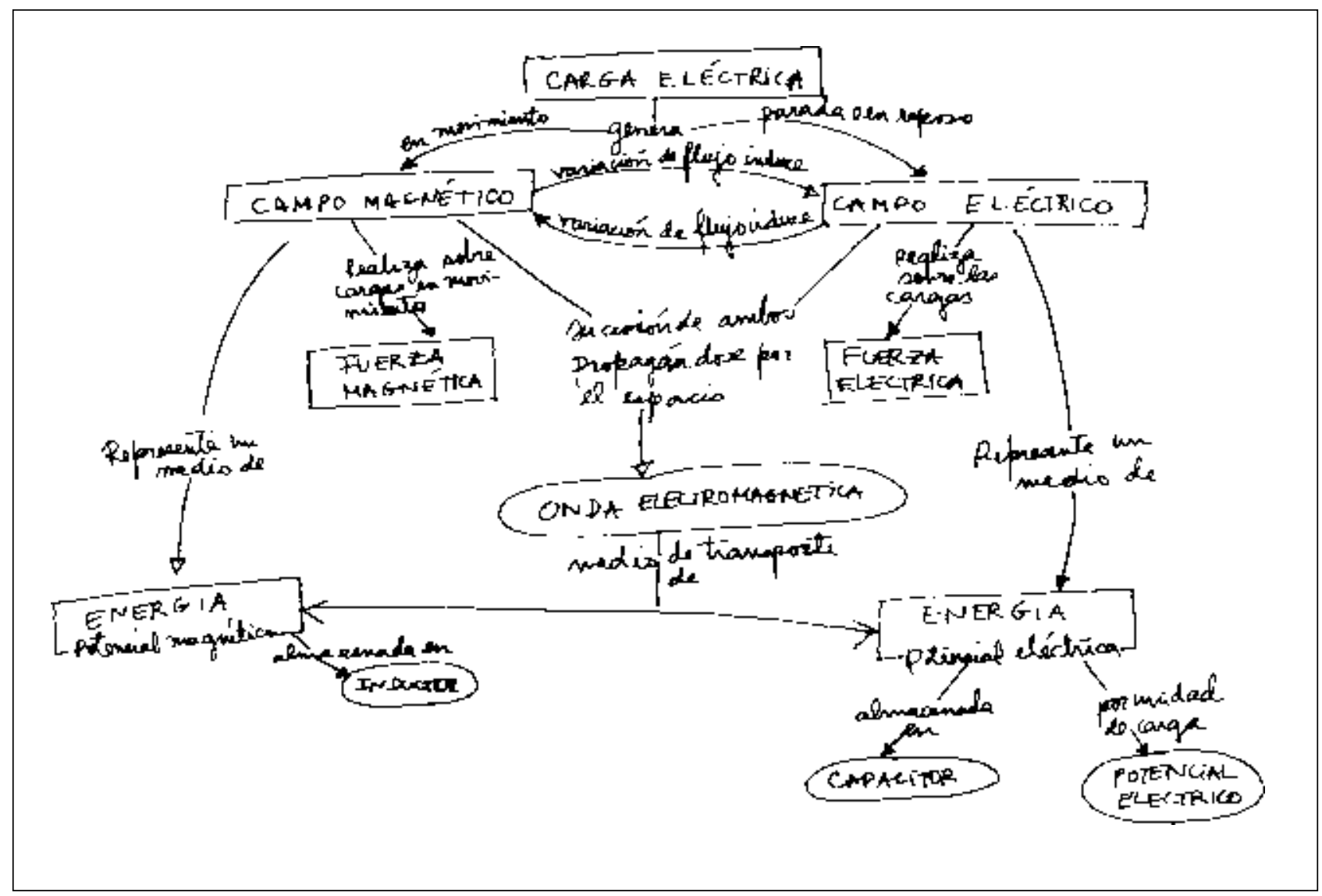

